\title{
Performance of the R-way Colposcopic Evaluation System in Cervical Cancer Screening
}

\author{
Jian Zhao' ${ }^{1}$ Xi Zhang ${ }^{2}$, Rui Chen ${ }^{3}$, Yu-Qian Zhao ${ }^{2}$,Ting-Ting Wang ${ }^{4}$, Shan $\mathrm{He}^{5}$, \\ You-Lin Qiao ${ }^{2 *}$
}

\begin{abstract}
Objective: To investigate the diagnostic value of the R-way colposcopic evaluation system (R-way system) in cervical cancer screening. Materials and Methods: Between August 2013 and August 2014, a total of 1,059 cases referred to colposcopy in Peking University First Hospital were studied using both the R-way system and conventional colposcopy. Our study evaluated and compared the diagnostic ability of the two methods in detecting high-grade lesions and cervical cancer (hereinafter called CIN2+). Evaluation indicators including sensitivity, specificity, positive predictive value (PPV), negative predictive value (NPV), Youden index and the area under the curve (AUC) of the receiver operating characteristic (ROC) were calculated. Results: The R-way system had a slightly lower specificity $(\mathbf{9 4 . 5 \%})$ than conventional colposcopy $(\mathbf{9 6 . 0} \%)$ for CIN2+ detection $(P=0.181)$. However, the sensitivity (77.8\%) was significantly higher than with the conventional colposcopic method (46.6\%) $\left(\chi^{2}=64.351, P<0.001\right)$. In addition, the AUC of the ROC for CIN2+ detection using the R-way system $(0.839)$ was larger than that with conventional colposcopy $(0.731)(Z=4.348, P<0.001)$. If preliminary result had been drawn from cervical exfoliated cytology before colposcopy referral, combination of the R-way system with cytology could increase the sensitivity to $93.9 \%$ for CIN2+ detection (excluding ASCUSLSIL), confirmed by multipoint biopsy or ECC. Conclusions: The diagnostic value of the R-way evaluation system is higher than that of conventional colposcopic evaluation in cervical cancer screening. Moreover, taking the ease of use and standardized quality control management into account, the $\mathbf{R}$-way system is highly preferable.
\end{abstract}

Keywords: R-way colposcopic evaluation system - conventional colposcopic examination - cervical cancer screening

Asian Pac J Cancer Prev, 16 (10), 4223-4228

\section{Introduction}

Cervical cancer is one of the most common cancers in women globally, with the fourth highest worldwide mortality. In less developed countries, cervical cancer is the second most prevalent cancer, with the second highest mortality in women among all other cancers. In developed countries, effective cervical cancer screening has lead to the morbidity and mortality to drop significantly. Now cervical cancer is no longer one of the top ten cancers in developed countries (Sigel et al., 2014; Torre et al., 2015).

In China, due to the large population and the lack of effective cytology interpretation system, a populationbased screening of high-risk HPV test is more suited for first-line screening (Qiao et al., 2008). Population screening is the secondary means of prevention and treatment of cervical cancer. For China to improve the quality of population screening, a feasible strategy would be to raise the level of colposcopy techniques of gynecologists. For cervical cancer screening, it is important that gynecologists practice a more standardized colposcopic evaluation system, especially for those in primary hospitals. R-way (R: Red; w: white; a: abnormal vascular features; y: yellow) is a standardized colposcopic evaluation system based on standard colposcopy procedure. By combining the sequentially appeared features ("R", "w", "a", “y"), a preliminary diagnostic result will be drawn along with a suggestion of biopsy regions, if required. R-way system is built to help gynecologists easily locate CIN2+ lesions, providing technical assurance for decreasing the morbidity and mortality of cervical cancer in China.

\section{Materials and Methods}

\section{Clinical materials}

Between 28 $8^{\text {th }}$ August 2013 and 28 ${ }^{\text {th }}$ August 2014, a total number of 1059 cases referred to colposcopy in Peking University First Hospital, were studied using both R-way system and conventional colposcopic method. Excluded criteria were: $i$ ) age of 25 years or less, $i$ i) a history of hysterectomy, iii) a history of pelvic radiation therapy. Colposcopy is performed on non-pregnancy

Department of Gynaecology and Obstetrics, ${ }^{1}$ Peking University First Hospital, Peking University Health Science Center, Peking University, ${ }^{3}$ Beijing Tsinghua Changguang Hospital, ${ }^{5}$ Beijing Luhe Hospital, Capital Medical University, ${ }^{2}$ Department of Cancer Epidemiology, National Cancer Center, Cancer Hospital, Chinese Academy of Medical Sciences \& Peking Union Medical College ${ }^{4}$ Chinese Center For Disease Control And Prevention, Beijing, China*For correspondence: qiaoy@cicams.ac.cn. 
women with a diagnosis of abnormal cervical exfoliated cytology, positive high-risk type HPV16/18 or suspicious for cervical lesions. Biopsy is conducted if either method suggests pathology. For all cases studied, histopathological diagnostic result of the cervix is included. The age of all studied cases was between 25-85 years old, with a mean age of $42.61 \pm 10.13$ years.

\section{Research methods}

Conventional colposcopy: According to standard colposcopy procedure, first saline is applied to clean the surface of the cervix, followed by preliminary inspection of the cervical surface. After that, $5 \%$ diluted acetic acid solution is applied to wipe the cervix for 50 seconds. If acetowhite epithelium occurs after application of acetic acid, images are captured at time points of $60 \mathrm{~s}, 90 \mathrm{~s}, 120 \mathrm{~s}$, observe the changes of the whitening effect during this period, check any abnormal vascular features such as punctuation, mosaics and atypical vessels. Finally, Lugol's staining (Schiller's test) is performed to inspect iodine uptake. From the information collected during this period, one of the four preliminary diagnostic results including suspicious for invasion, high-grade lesions, low-grade lesions and normal colposcopic findings (Quaas et al., 2014) can be drawn. For the cases in which colposcopic examination did not indicate suspicious cervical cancer or a high-grade lesion but cervical cytology showed a positive diagnostic result of ASC-H, HSIL, SCC, AGC+ (Including AGC-NOS and AGC-FN), AIS or Adca, multipoint biopsy or ECC was conducted afterwards. Collected tissue is fixed with $10 \%$ formalin and examined in pathological department.

R-way system: Supplemental instructions and materials were added into conventional colposcopy method: First, saline is applied to clean the surface of the cervix followed by inspection of surface vessels under colposcope. Current images are captured with and without green filter to evaluate the "Red" feature. If "red" is obvious, mark the corresponding region in a quadrantal diagram (Figure 1 ) on the computer. After that, $5 \%$ diluted acetic acid is applied to wipe the cervix for 50 seconds. The changes of the surface of the cervix are observed continuously and images are captured at $60 \mathrm{~s}, 90 \mathrm{~s}, 120 \mathrm{~s}$. The whitening effect is inspected from these 3 images to see whether the acetowhite epithelium fades or becomes dense and thick. If acetowhite epithelium persisted or incrassated, this feature is defined as "White" and the corresponding area should be marked in a quadrantal diagram (Figure 2). Then checking whether punctuation, mosaics and atypical vessels (a) appear in acetowhite areas, mark the corresponding region in a quadrantal diagram (Figure 3 ). Finally, Lugol's iodine solution is applied to the cervix to see the region with no iodine uptake. Mark the "Mustard Yellow" region (y) with no iodine uptake (Figure 4). Based on different combination of above marks, the computer associates and compares the images captured during colposcopic examination, performs analysis using the pathology-based R-way specialized database, then provides preliminary diagnosis (suspicious for invasion, high-grade lesions, low-grade lesions or normal colposcopic findings) and suggests biopsy regions for cases of suspicious for invasion or high-grade lesions. If $\mathrm{R}$-way system failed to recognize cases of suspicious for
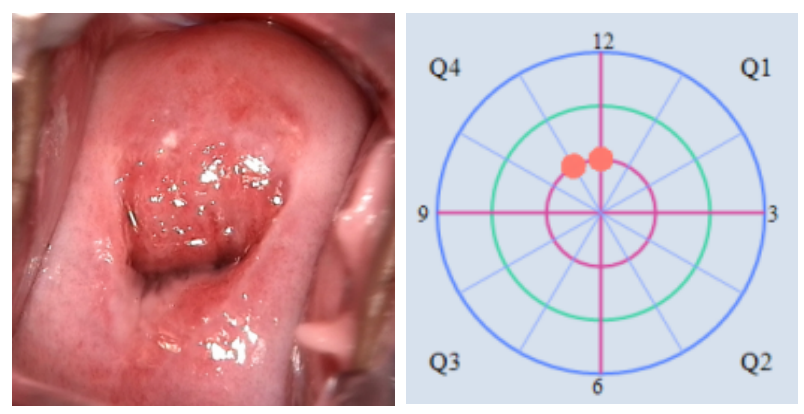

Figure 1. PLEASE SUPPLY
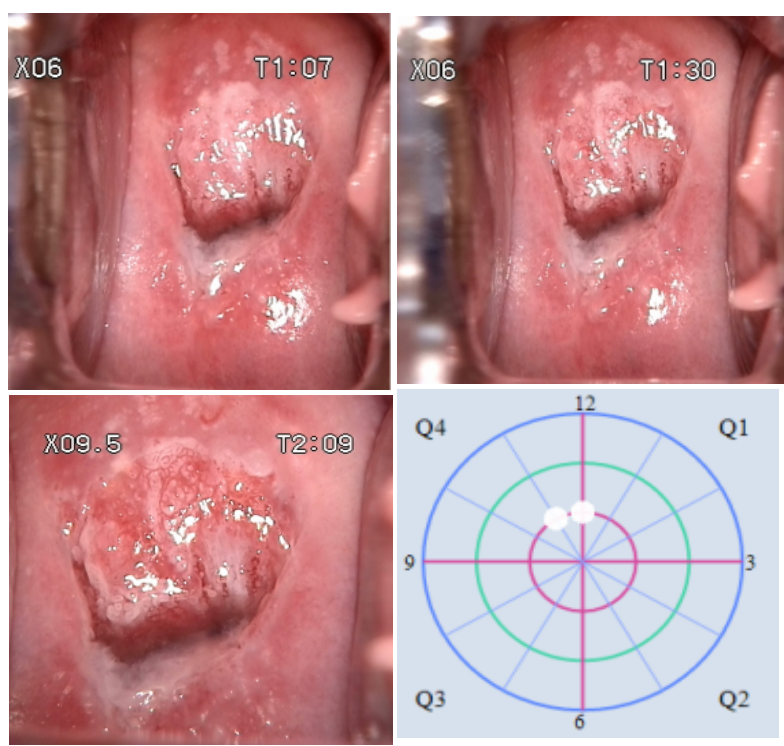

Figure 2. PLEASE SUPPLY
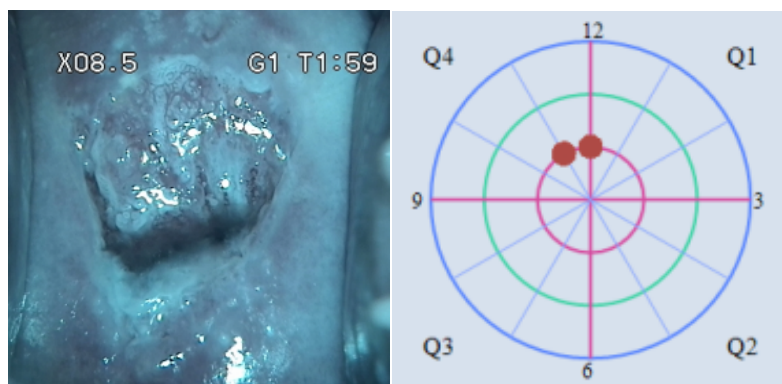

Figure 3. PLEASE SUPPLY
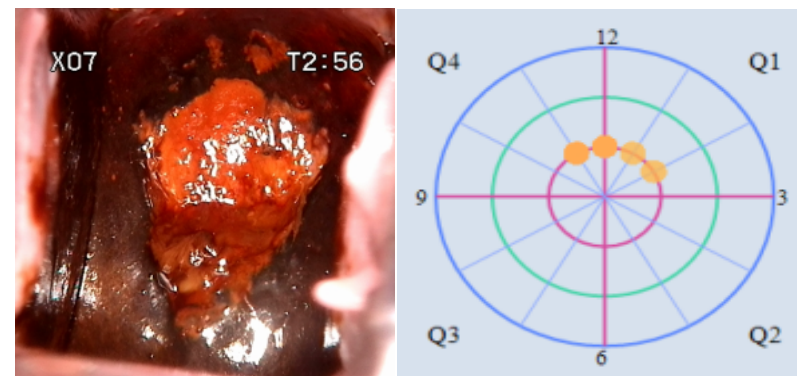

Figure 4. PLEASE SUPPLY 
Table 1. Results of Two Different Methods for Detection of CIN2 and Higher Lesions

\begin{tabular}{|c|c|c|c|c|c|c|}
\hline \multirow[t]{2}{*}{ Evaluating indicator } & \multirow{2}{*}{$\begin{array}{c}\text { R-way system } \\
\%\end{array}$} & \multicolumn{3}{|c|}{ Conventional colposcopy } & \multirow[t]{2}{*}{$\chi^{2}$} & \multirow[t]{2}{*}{$p$} \\
\hline & & $95 \%$ C.I. & $\%$ & 95\% C.I. & & \\
\hline \multicolumn{7}{|c|}{ Not combined with cytology } \\
\hline Sensitivity & 77.81 & $72.87,82.08$ & 46.62 & $41.15,52.18$ & 64.351 & $<0.001$ \\
\hline Specificity & 94.52 & $92.65,95.93$ & 95.99 & $94.33,97.18$ & 1.789 & 0.181 \\
\hline PPV & 85.51 & $80.94,89.14$ & 82.86 & $76.58,87.72$ & 0.582 & 0.446 \\
\hline NPV & 91.11 & $88.9,92.91$ & 81.22 & $78.51,83.66$ & 33.237 & $<0.001$ \\
\hline Youden index & 72.33 & - & 42.61 & - & - & - \\
\hline \multicolumn{7}{|c|}{ Combined with cytology } \\
\hline Sensitivity & 93.89 & $90.66,96.05$ & 58.84 & $53.3,64.17$ & 105.836 & $<0.001$ \\
\hline Specificity & 89.71 & $87.32,91.68$ & 92.51 & $90.4,94.19$ & 3.639 & 0.056 \\
\hline PPV & 79.13 & $74.7, \quad 82.97$ & 76.57 & $70.8, \quad 81.49$ & 0.558 & 0.455 \\
\hline NPV & 97.25 & $95.74,98.23$ & 84.39 & $81.75,86.71$ & 70.477 & $<0.001$ \\
\hline Youden index & 83.6 & - & 51.36 & - & - & - \\
\hline
\end{tabular}

Table 2. Comparison of the AUC of the ROC Assessed by R-way System or Conventional Method

\begin{tabular}{lcccc}
\hline Method & $\begin{array}{c}\text { R-way } \\
\text { system }\end{array}$ & $\begin{array}{c}\text { Conventional } \\
\text { method }\end{array}$ & $Z$ & $P$ Value \\
\hline Not combined with cytology & & & \\
AUC* & 0.839 & 0.731 & 4.348 & $<0.001$ \\
95\% CI & $0.807-0.871$ & $0.693-0.768$ & \\
Combined with cytology & \multicolumn{4}{c}{} \\
AUC* & 0.923 & 0.79 & 6.743 & $<0.001$ \\
95\% CI & $0.904-0.942$ & $0.756-0.823$ & \\
\hline *Null hypothesis: AUC $=0.5, P<0.001$ & & &
\end{tabular}

invasion or high-grade lesions, R-way will automatically correlate these two situation below for biopsy: $i$ ) If blood bleeding occurs on the surface of the cervix (or ulceration), bleeding surface biopsy or ulceration biopsy is suggested; ECC is recommended if blood bleeding is from cervical canals. ii) Biopsy of random region in four quarters or ECC is suggested if cervical cytology result is ASC-H, HSIL, SCC, AGC+ (AGC-NOS and AGC-FN included), AIS or Adca. Collected tissue is fixed with $10 \%$ formalin and examined in the pathological department.

\section{Preliminary colposcopy results}

Preliminary results from colposcopy are classified into four groups including: suspicious for invasion, highgrade lesions, low-grade lesions and normal colposcopic findings. Preliminary results of normal colposcopic findings and low-grade lesions are grouped as negative. Results of suspicious for invasion and high-grade lesions are classified as positive by using conventional colposcopic method while active bleeding is also considered a positive group when using the R-way system. Among all cases, when suspicious for invasion or high-grade lesions is not recognized by colposcopy while cervical cytology shows a diagnostic result of ASC-H, HSIL, SCC,AGC+ (including AGC-NOS and AGC-FN), AIS or Adca, those cases are classified as positive enrolling in statistics analysis.

\section{Pathology examination and results interpretation}

Histopathology result is regarded as the gold standard in this study. According to American Society for Colposcopy Cervical Pathology (ASCCP) guidelines published in 2013 (Massad et., 2013), pathological findings are classified into four levels, namely cervicitis,
LSIL (CIN I), HSIL (CIN II-III) and invasive cancer. Pathological negative group refers to the low-grade lesions group, including cervicitis, LSIL (CIN I) and HPV relevant lesions. Pathological positive group refers to high-grade lesions group including HSIL, adenocarcinoma in situ and invasive cervical cancer.

\section{Statistics}

All statistical analyses have been conducted using SPSS 17.0 software. Evaluation the ability of two methods on detecting CIN2+ is performed by analyzing the sensitivity, specificity, positive predictive value (PPV), negative predictive value (NPV) and Youden index. The diagnostic ability of these two methods is assessed by comparing the AUC of the ROC. We respectively calculated chi-square test and $\mathrm{Z}$ test for comparisons between evaluation indicators of two methods. $P$ values less than 0.05 is defined as significantly different.

\section{Results}

\section{Evaluating indictors}

Among 1059 cases enrolled in this study, 311 cases are diagnosed of cervical diseases by gold standard while the other 748 cases are confirmed normal. R-way system shows a positive result of 242 cases (292 cases when combined with cytology) while conventional method reports 145 positive cases (183 cases when combined with cytology). The sensitivity, PPV, NPV and Youden index of R-way system $(77.81 \%, 85.51 \%, 91.11 \%, 72.33 \%)$ are higher than those of conventional method (46.62\%, $82.86 \%, 81.22 \%, 42.61 \%)$ for CIN2 + detection. The specificity of R-way system (94.52\%) is slightly lower than that of conventional colposcopic method (95.99\%), but the difference Is not statistically significant $(\mathrm{P}=0.181)$. (Table 1).

\section{AUC of the ROC}

Receiver operating characteristics (ROC) curves are plotted to evaluate the sensitivity and specificity of conventional colposcopy and R-way system for CIN2+ detection. AUC above 0.5 is defined as clinical significant. From the results, two methods tested are clinical significant for $\mathrm{CIN} 2+$ detection $(\mathrm{P}<0.001)$. The AUC of the R-way system is 0.839 (95\% CI: 0.807-0.871), 


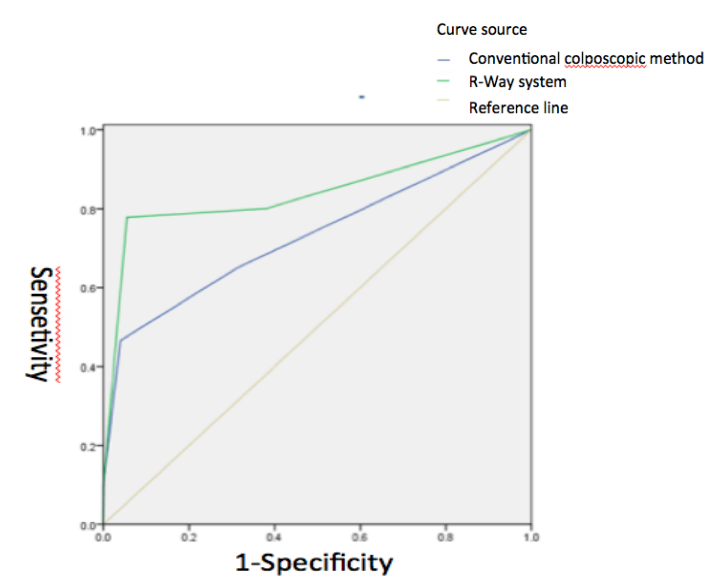

Figure 5. Comparison of the AUC of the ROC Assessed by R-way System and Conventional Method without Combination of Cytology

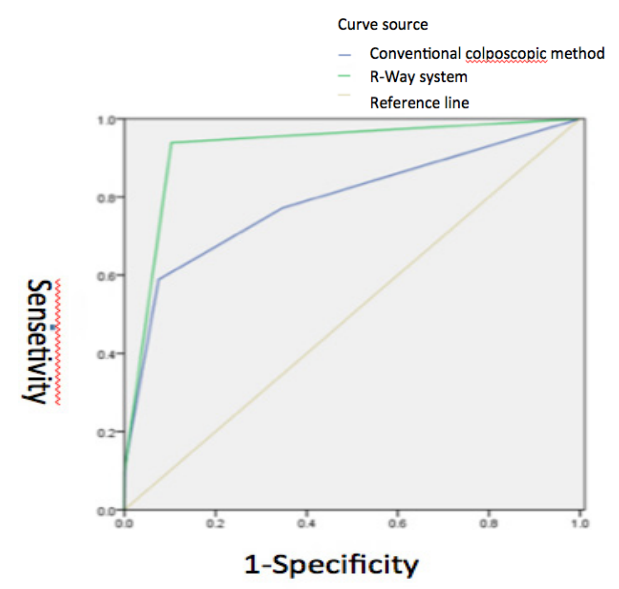

Figure 6. Comparison of the AUC of the ROC Assessed by R-way System or Conventional Method with Combination of Cytology

while the AUC of conventional colposcopic method is 0.731 (95\% CI: 0.693-0.768), The AUC of the ROC assessed by two different method is significant different $(\mathrm{P}<0.001)$. Therefore, $\mathrm{R}$-way system performs better than conventional colposcopic method for CIN2+ detection with regard to the AUC (Table 2, Figure 5, Figure 6).

\section{Discussion}

Cervical cancer is one of the most common malignant tumors in women's genital tracts. Early diagnosis and treatment of cervical premalignant lesions is very important for the prognosis of cervical cancer. To effectively reduce the morbidity and mortality of cervical cancer, women at the right age are injected with HPV vaccine and cervical cancer screening campaigns are conducted in large scale. In China, HPV vaccine has not yet approved by CFDA, hence cervical cancer screening remains an important way for cervical cancer prevention (Petry et al., 2013). In 2008 the Chinese government launched 'Breast cancer \& Cervical cancer' examination project for women in rural areas., In three years over 10 million women have been screened. In 2012, the coverage of the project increased to 10 million cases per year.
The primary method of cervical cancer screening is to apply Papanicolaou smear or VIA/VILI (Cristiani et al., 2014), and patients with positive diagnosis would be then referred to colposcopy[9]. As the second stage for cervical cancer screening, colposcopy goes as follows: observe the images of the cervical surface (which indirectly reflect the components of cervical tissues) to identify CIN2+ by finding whether abnormal cells and vessels present on the surface of the cervix. This could improve biopsy accuracy, avoid excessive biopsy. Pathological biopsy under colposcope is the gold standard for determining whether treatment is needed or not. Therefore, the precise location of CIN2+ region is not only the assurance of a proper pathological diagnosis, but also a critical technical problem encountered in cervical cancer screening. However, image interpretation is dominated by subjective experience of gynecologist during conventional colposcopy (Thulaseedharan et al., 2015), with the sensitivity in detecting High-grade Squamous Intraepithelial Lesion (HSIL) ranges from $48 \%$ to $87 \%$ in different reports (Di Stefano et al., 2014; Diedrich et al., 2014; Ghosh et al., 2014; Tatiyachonwiphut et al., 2014; Thulaseedharan et al., 2015). Based on pathology, R-way system identifies cases of high-grade lesions or suspicious for invasion by observing the staining effects of cervical epithelium after application of different solutions. Abnormal cervical tissues consist of abnormal newly-formed vessels and abnormal cells. If large amount of newly-formed vessels and abnormal cells present on the surface of the cervix, terminal cervical cancer can be detected through visual inspection of changes such as cervical ulceration, necrosis, bleeding, irregular surface, or ectocervical dysplastic nodules in routine gynecological examinations. The target group for cervical cancer screening are patients with HSIL. Therefore, early detection of HSIL is the primary task of colposcopy. HSIL is diagnosed with colposcopy rather than naked eyes when newly-formed vessels and abnormal cells are not sufficient for visual inspection. During colposcopy, by applying 5\% dilute acetic acid solution and Lugol's solution (Schiller's test), images were captured by colpocscope for further analysis. Those images were formed by the light which reflected from the stroma underlying the epithelial cells. By observation and analysis of image features such as the colour, shape, border and vessels, HSIL could be detected.

In normal cases, the cervical columnar epithelium is composed of a single layer of glandular cells and it appears reddish in colour because the thin single cell layer makes the underlying vasculature in the stroma easier to be seen. Due to multiple factors, reserve cells under the columnar epithelium start to become squamous. At the beginning of this process, the underlying vasculature in the stroma also appears reddish in immature cervical transformation zones (TZ). When the cervix goes atrophic, differentiation of the intermediate and superficial cell layers becomes slower due to hormone level decreased, and usually only the bottom layer of squamous cells is visible. Therefore, the reddish feature appears again in atrophic cervical areas.

One typical feature of abnormal cells is the higher nuclear density compared with normal cells. $5 \%$ dilute acetic acid solution reacts with the nucleus protein of 
abnormal cells in HSIL region, leading to a reduction of transmittance. While the light can reflect back from the stroma after passing through the epithelial cell layers of normal cervical tissues, forming acetowhite pattern in contrast with the abnormal tissues. Special attention should be paid on another variant of HSIL, thin HSIL, with cell layers less than 10 (Darragh et al., 2013). Under this circumstances, transmittance of the tissue is only reduced slightly, together with no thick acetowhitening effects. In this case, the time length of whitening effects and vessels in acetowhite areas are critical in distinguishing abnormality from normality.

Appearance of abnormal cells is always accompanied with new vessels formation. During expansion, the newly-formed vessels are squeezed by abnormal cells and appear irregular in the acetowhite epithelium, and the commonly-seen abnormalities are coarse punctation, coarse unsymmetrical mosaics and other atypical vessels.

Lugol's solution stains cells with abundant glycogen. Normally, in the cervix, there is little glycogen present in columnar epithelial cells, immature metaplastic cells and the intermediate and bottom layers of squamous epithelial cells, whereas glycogen is rich in original and newly formed mature squamous epithelium. Therefore, in 2011 IFCPC colposcopic nomenclature (Bornstein et al., 2012), Lugol's staining (Schiller's test) was categorized as a non-specific staining test, and it is recommended that Lugol's staining should be performed on the acetowhite epithelium during colposcopy.

Based on standardized time control of the examination procedure and observation of changes analyzed by the computer, the R-way system could automatically identify CIN2+. With the R-way system, it shows a higher sensitivity $(77.81 \%)$ than the conventional colposcopic method does (with a sensitivity of $46.62 \%$ ) in identifying CIN2+. If further combined the R-way system with cytology, the sensitivity could reach to $93.89 \%$. With the R-way system, the AUC of the ROC curve reaches 0.839 (95\% CI: 0.807-0.871). All these indicate that the R-way system performs better than the conventional colposcopic method in identifying CIN2+ lesions.

All technology has its limitations, and colposcopy is no exception. The R-way system is strictly to comply with the pathological features of cervical lesions. The R-way system would suggest a biopsy from the cervical bleeding area or endocervical bleeding area for patients detected with the presence of abnormal newly-formed vessels and abnormal cells under colposcope, especially for those with bleeding (or ulceration) seen on the cervical surface or endocervical canal after application of 5\% diluted acetic acid solution. For patients with cytological report of ASC-H, HSIL, SCC, AGC+ (including AGC-NOS and AGC-FN), AIS or Adca, multipoint biopsy or ECC is recommended.

In America, professional colposcopists need to know the techniques of colposcopy and also, the knowledge of relevant subjects of lower genital tract, including the pathophysiology, cytology, cytogenetics, preventive medicine, basic research, molecular biology, gynecological oncology and gynecological endocrinology. In low resource countries, it is difficult to elevate the levels of primary gynecologist in a short period, thus enhancing the accuracy of CIN2+ detection is the current approach. With R-way system, colposcopy is better at detection of CIN2+.To complete a colposcopy the colposcopist needs to know: how to use the relevant equipiment, standard colposcopy procedure, identification of the colour features (red, white and yellow) after application of the three examination solutions, and the use of a computer. Another factor deals with the colposcopist's experience. For most colposcopists, especially primary colposcopist in rural areas, the accuracy and consistency of preliminary results varied from case to case. During colposcopy, the operator identifies the colour features in each step and marks them in real-time if required via the R-way system. Then the computer associates and compares images captured, performs analysis using the pathologybased R-way specialized database, and a more objective result will be addressed in this manner. Through simple training, primary colposcopist can quickly grasp the use of the R-way system. This easy-to-use system provides a basis for a more accurate diagnosis by diminishing subjective judgment, hence it is of great importance for enhancing the efficiency of cancer screening, especially in low resources countries.

In conclusions, the R-way system performs better than the conventional colposcopic method in identifying high-grade cervical lesions. Through time control of colposcopy procedure, the R-way system standardizes the time points for each images captured, identifies the colour changes (red, white and yellow) in each step and evaluates the presence of transformation zone. For its ease of use and standardized management of colposcopy procedure, R-way system is highly recommended for cervical cancer screening.

\section{Acknowledgements}

The research was fund by Organization of Cervical Cancer Prevention Facility in China from International Agency for Research on Cancer (IARC).

\section{References}

Bornstein J, Bentley J, Bosze P, et al (2012). 2011 Colposcopic terminology of the international federation for cervical pathology and colposcopy. Obstet Gynecol, 120, 166-72.

Cristiani P, Costa S, Schincaglia P, et al (2014). An online quality assurance program for colposcopy in a population-based cervical screening setting in Italy: results on colposcopic impression. J Low Genit Tract Dis, 18, 309-13.

Darragh TM, Colgan TJ, Thomas Cox J, et al (2013). The lower anogenital squamous terminology standardization project for HPV-associated lesions: background and consensus recommendations from the college of American pathologists and the American society for colposcopy and cervical pathology. Int J Gynecol Pathol, 32, 76-115.

Di Stefano L, Patacchiola F, Necozione S, et al (2014). The correspondence between abnormal transformation zone grade 1 and grade 2 colposcopic parameters and histology. clinical implications. Eur J Gynaecol Oncol, 35, 16-9.

Diedrich JT, Felix JC, Lonky NM (2014). Contribution of exocervical biopsy,endocervical curettage, and colposcopic grading in diagnosing high-grade cervical intraepithelial 


\section{Jian Zhao et al}

neoplasia. J Low Genit Tract Dis, 18, 1-7.

Ghosh I, Mittal S, Banerjee D, et al (2014). Study of accuracy of colposcopy in VIA and HPV detection-based cervical cancer screening program. J Obstet Gynaecol, 54, 570-5.

Massad LS, Einstein MH, Huh WK, et al (2013). 2012 ASCCP consensus guidelines conference. 2012 updated consensus guidelines for the management of abnormal cervical cancer screening tests and cancer precursors. J Low Genit Tract Dis, 17, 1-27.

Petry KU, Luyten A, Scherbring S, et al (2013). Accuracy of colposcopy management to detect CIN3 and invasive cancer in women with abnormal screening tests: results from a primary HPV screening project from 2006 to 2011 in Wolfsburg, Germany. Gynecol Oncol, 128, 282-7.

Qiao YL, Sellors JW, Eder PS, et al (2008). A new HPV-DNA test for cervical-cancer screening in developing regions: a cross-sectional study of clinical accuracy in rural China. Lancet Oncol, 9, 929-36

Quaas J, Reich O, Kuppers V (2014). Explanation and use of the colposcopy terminology of the IFCPC (international federation for cervical pathology and colposcopy) Rio 2011,Geburtshilfe und Frauenheilkunde, 74, 1090-2.

Sigel R, Ma JM, Zou ZH, et al (2014). Cancer Statistics, 2014. Cancer J Clin, 64, 9-29.

Tatiyachonwiphut M, Jaishuen A, Sangkarat S, et al (2014). Agreement between colposcopic diagnosis and cervical pathology: Siriraj hospital experience. Asian Pac J Cancer Prev, 15, 423-6.

Thulaseedharan JV, Malila N, Esmy PO, et al (2015).Risk of invasive cancer among women visually screened and colposcopy triaged by trained nurses in rural south India. Int J Gynaecol Obstet [Epub ahead of print].

Torre LA, Bray F, Siegel RL, et al (2014). Global cancer statistics, 2012. CA Cancer J Clin [Epub ahead of print]. 